\title{
On class visualisation for high dimensional data: Exploring scientific data sets
}

\author{
Ata Kabán ${ }^{1}$, Jianyong Sun ${ }^{1,2}$, Somak Raychaudhury ${ }^{2}$ and Louisa Nolan ${ }^{2}$ \\ ${ }^{1}$ School of Computer Science ${ }^{2}$ School of Physics and Astronomy \\ The University of Birmingham, Birmingham, B15 2TT, UK \\ \{axk,jxs\}@cs.bham.ac.uk, \{somak, lan\}@star.sr.bham.ac.uk
}

\begin{abstract}
Parametric Embedding (PE) has recently been proposed as a general-purpose algorithm for class visualisation. It takes class posteriors produced by a mixture-based clustering algorithm and projects them in $2 \mathrm{D}$ for visualisation. However, although this fully modularised combination of objectives (clustering and projection) is attractive for its conceptual simplicity, in the case of high dimensional data, we show that a more optimal combination of these objectives can be achieved by integrating them both into a consistent probabilistic model. In this way, the projection step will fulfil a role of regularisation, guarding against the curse of dimensionality. As a result, the tradeoff between clustering and visualisation turns out to enhance the predictive abilities of the overall model. We present results on both synthetic data and two real-world high-dimensional data sets: observed spectra of early-type galaxies and gene expression arrays.
\end{abstract}

\section{Introduction}

Clustering and visualisation are two widespread unsupervised data analysis techniques, with applications in numerous fields of science and engineering. Two key strategies can be distinguished in the literature: (1) One is to produce a compression of the data first and then use that to visually detect distinct clusters. A wide range of linear and nonlinear dimensionality reduction techniques developed in machine learning follow this route, including PCA, GTM [4, etc. (2) The alternative strategy is to cluster the data first and visualise the resulting cluster assignments afterwards. This is more popular in the data mining community [13]. A recently proposed method, termed Parametric Embedding (PE) [7 proposes to take class posteriors produced by a mixture-based clustering algorithm and project them in $2 \mathrm{D}$ for visualisation.

Let us observe however, that for both of these data exploration strategies the two objectives - clustering and visualisation - are decoupled and essentially one is entirely subordinated to the other. This is worrying in that inevitably, the errors accumulated in the first stage cannot be corrected in a subsequent stage and may essentially compromise the process of understanding the data.

In this paper we consider the class visualisation problem, as in [7] and we identify a setting where a more fruitful coupling between clustering and visualisation can be achieved by integrating them both into a consistent probabilistic 
model. As we shall see, this is particularly useful in high-dimensional problems, where the number of data dimensions exceeds the number of observations. Such cases are encountered in modern scientific data analysis, e.g. in gene expression analysis, or the analysis of special objects in astronomy.

Our approach is based on a multi-objective formulation in a probabilistic formalism. As we shall see, our model can be decoupled and understood as a sum of two objectives: One term is responsible for clustering and one other for a PE-like projection of the estimated class assignments. These two steps are now interdependent, so that in high dimensional problems the projection step fulfils a regularisation role, guarding against the curse of dimensionality problem.

We use both synthetic data and two real-world high-dimensional data sets in our experiments: Observed spectra (of rare quality and coverage) of early-type galaxies and a benchmark gene expression array data set are used to demonstrate the working of the proposed approach. We find that in both cases we obtain not only a visualisation of the mixture posteriors, but a more predictive mixture model, as measured by out of sample test likelihood, as a result of appropriately combining the objectives of clustering and class projection.

The remainder of the paper is organised as follows: We begin with presenting our probabilistic model in Section 2. The interpretation by which this can be understood as a joint model for mixture based clustering and PE-like projection will become evident in Section 3, where the EM 3] methodology is used to derive a maximum a posteriori (MAP) estimation algorithm for our model. We then extend this work to take into account additional available knowledge on measurement uncertainties for real-world data analysis. Section 4 presents the application two very different experiments involving galaxy spectra and gene expression. The results are summarised in the concluding section.

\section{The model}

Consider $N$ independent, $T$-dimensional data points. The $n$-th point is denoted by $\boldsymbol{d}_{n} \in \mathcal{R}^{T \times 1}$ having features $d_{t n}$. We seek a $2 \mathrm{D}$ mapping of this data into points $\boldsymbol{x}_{n} \in \mathcal{R}^{2 \times 1}$ in the Euclidean space such as to reflect topological relationships based on some cluster structure that may be present in the data set. In building up our model, we begin by making the common assumption of conditional independence, in order to enforce the dependences among data features to be captured in the latent space.

$$
p\left(\boldsymbol{d}_{n} \mid \boldsymbol{x}_{n}\right)=\prod_{t} p\left(d_{t n} \mid \boldsymbol{x}_{n}\right)
$$

Further, in order to capture complicated density shapes, including possibly distinct clusters, we model the conditional probabilities of the data features as a mixtures of Gaussians. The mixing coefficients of these mixtures are instancespecific, so that each measurement that belongs to the same object will have the same mixing coefficient. This will ensure that the various features of an instance 
are likely to be allocated to the same (set of) mixture components.

$$
p\left(d_{t n} \mid \boldsymbol{x}_{n}\right)=\sum_{k} p_{\theta_{t k}}\left(d_{t n} \mid k\right) P_{\boldsymbol{c}_{k}}\left(k \mid \boldsymbol{x}_{n}\right)
$$

Observe we do not impose that each data point must belong to exactly one mixture component. This allows us to model the relationships between clusters.

Assuming that we work with real-valued observations, and $p\left(d_{t n} \mid k\right)$ is a Gaussian, then $\theta_{t k}=\left\{\mu_{t k}, v_{t k}\right\}$ are the mean and precision parameters respectively. Other choices are however possible as appropriate.

$$
p\left(d_{t n} \mid k\right)=\mathcal{N}\left(d_{t n} \mid \mu_{t k}, v_{t k}\right)
$$

The second factor in (2) is a nonlinear function that projects a point $\boldsymbol{x}_{n}$ from the Euclidean space onto a probability simplex. A parameterised softmax function can be used for this purpose.

$$
P_{\boldsymbol{c}_{k}}\left(k \mid \boldsymbol{x}_{n}\right)=\frac{\exp \left\{-\frac{1}{2}\left(\boldsymbol{x}_{n}-\boldsymbol{c}_{k}\right)^{2}\right\}}{\sum_{k^{\prime}} \exp \left\{-\frac{1}{2}\left(\boldsymbol{x}_{n}-\boldsymbol{c}_{k^{\prime}}\right)^{2}\right\}}
$$

Our goal is then to determine $\boldsymbol{x}_{n}$ for each $\boldsymbol{d}_{n}$. In addition, we also need to estimate the parameters $\theta_{t k}$ and $\boldsymbol{c}_{k}$.

In order to somewhat narrow down the search space, we add smoothing priors, similarly to []:

$$
\boldsymbol{x}_{n} \sim \mathcal{N}\left(\boldsymbol{x}_{n} \mid \mathbf{0}, \alpha \boldsymbol{I}\right) ; \boldsymbol{c}_{k} \sim \mathcal{N}\left(\boldsymbol{c}_{k} \mid \mathbf{0}, \beta \boldsymbol{I}\right)
$$

In addition, the inverse variance parameters (precisions) are given exponential priors to prevent them produce singularities and encourage the extinction of unnecessary model complexity.

$$
p\left(v_{t k}\right)=\gamma e^{-\gamma v_{t k}}
$$

The hyperparameters $\alpha, \beta$ and $\gamma$ must all have strictly positive values.

\section{Parameter estimation}

Here we derive MAP estimates for our model specified in the previous section. The complete data log likelihood is proportional to the posterior over all hidden variables and this is the following.

$$
\begin{aligned}
\mathcal{L} & =\sum_{n, t} \log \sum_{k} p_{\theta_{t n}}\left(d_{t n} \mid k\right) P \boldsymbol{c}_{k}\left(k \mid \boldsymbol{x}_{n}\right)+\sum_{k} \log P\left(\boldsymbol{c}_{k}\right)+\sum_{n} \log P\left(\boldsymbol{x}_{n}\right)+\sum_{t, k} \log P\left(v_{t k}\right) \\
& \geq \sum_{n} \sum_{t} \sum_{k} r_{k t n}\left\{\log p_{\theta_{t k}}\left(d_{t n} \mid k\right)+\log P \boldsymbol{c}_{k}\left(k \mid \boldsymbol{x}_{n}\right)-\log r_{k t n}\right\} \\
& +\sum_{n} \log P\left(\boldsymbol{x}_{n}\right)+\sum_{k} \log P\left(\boldsymbol{c}_{k}\right)+\sum_{t, k} \log P\left(v_{t k}\right)
\end{aligned}
$$


where we used Jensen's inequality and $r_{k t n} \geq 0, \sum_{k} r_{k t n}=1$ represent variational parameters that can be obtained from maximising (7):

$$
r_{k t n}=\frac{p_{\theta_{t k}}\left(d_{t n} \mid k\right) P_{\boldsymbol{c}_{k}}\left(k \mid \boldsymbol{x}_{n}\right)}{\sum_{k^{\prime}} p_{\theta_{t k}}\left(d_{t n} \mid k\right) P_{\boldsymbol{c}_{k}}\left(k \mid \boldsymbol{x}_{n}\right)}
$$

We can also regard $k=1, \ldots, K$ as the outcome of a hidden class variable and $r_{k t n}$ are in fact true class posterior probabilities of this class variable, cf. Bayes' rule.

The re-writing (7.8) is convenient for deriving the estimation algorithm for the parameters of the model. Before proceeding, let us rearrange (7) in two main terms, so that the interpretation of our model as a combination of mixture-based clustering and a PE-like class projection becomes evident.

$$
\begin{aligned}
\operatorname{Term}_{1} & =\sum_{n} \sum_{t} \sum_{k} r_{k t n} \log p_{\theta_{t k}}\left(d_{t n} \mid k\right)-\gamma \sum_{t, k} v_{t k}+\text { const. } \\
\operatorname{Term}_{2} & =\sum_{n} \sum_{t} \sum_{k} r_{k t n}\left\{\log P \boldsymbol{c}_{k}\left(k \mid \boldsymbol{x}_{n}\right)-\log r_{k t n}\right\}+\sum_{k} \log P\left(\boldsymbol{c}_{k}\right)+\sum_{n} \log P\left(\boldsymbol{x}_{n}\right) \\
& =\sum_{n, t}-K L\left(\boldsymbol{r}_{., t, n} \| P \boldsymbol{c} .\left(. \mid \boldsymbol{x}_{n}\right)\right)-\alpha \sum_{n}\|\boldsymbol{x}\|_{n}^{2}-\beta \sum_{k}\|\boldsymbol{c}\|_{k}^{2}+\text { const. }
\end{aligned}
$$

Now, the first term can be recognised as a clustering model, essentially an instance of modular mixtures [2] or an aspect-mixture of Gaussians 6 12], which is known to be advantageous in high-dimensional clustering problems 2]. The second term, in turn, is a PE-like objective [7, which minimises the KullbackLeibler (KL) divergence between the class-posteriors and their projections. Evidently, these two objectives are now interdependent. It remains to be seen in which cases their coupling is advantageous.

Carrying out the optimisation of (7) yields the following maximum likelihood estimates for the means and maximum a posteriori estimates for the precisions.

$$
\mu_{t k}=\frac{\sum_{n} r_{k t n} d_{t n}}{\sum_{n} r_{k t n}} ; v_{t k}=\frac{\sum_{n} r_{k t n}}{\sum_{n} r_{k t n}\left(d_{t n}-\mu_{t k}\right)^{2}+2 \gamma}
$$

For the remaining parameters, there is no closed form solution, we employ numerical optimisation using the gradients (see Appendix):

$$
\begin{aligned}
\frac{\partial}{\partial \boldsymbol{x}_{n}} & =\sum_{k}\left(\boldsymbol{c}_{k}-\boldsymbol{x}_{n}\right) \sum_{t}\left(r_{k t n}-P_{\boldsymbol{c}_{k}}\left(k \mid \boldsymbol{x}_{n}\right)\right)-\alpha \boldsymbol{x}_{n} \\
\frac{\partial}{\partial \boldsymbol{c}_{k}} & =\sum_{n}\left(\boldsymbol{x}_{n}-\boldsymbol{c}_{k}\right) \sum_{t}\left(r_{k t n}-P_{\boldsymbol{c}_{k}}\left(k \mid \boldsymbol{x}_{n}\right)\right)-\beta \boldsymbol{c}_{k}
\end{aligned}
$$

As expected, the form of parameter updates also reflects the interdependence of our two objectives: (11) is formally identical with the updates in 2612 (up to the variation implied by the use of the prior for precisions $\left.{ }^{1}\right)$. The gradients (12)-(13) are in turn, as expected, very similar to the updates in PE [7].

${ }^{1}$ In [12], the authors propose an improper prior for the variances. Incidentally, our MAP estimate for the precision parameter (11) is formally identical to the inverse of their variance estimates - so we now see the expression can be derived with the use of a proper exponential prior on the precisions. 


\subsection{Empirical Bayesian test likelihood}

Having estimated the model, the empirical Bayesian estimate [5] of the goodness of fit for new points is given by integrating over the empirical distribution $\frac{1}{N} \sum_{n} \delta\left(\boldsymbol{x}-\boldsymbol{x}_{n}\right)$. This is the following.

$$
p\left(\boldsymbol{d}_{\text {test }}\right)=\frac{1}{N} \sum_{n} p\left(\boldsymbol{d}_{\text {test }} \mid \boldsymbol{x}_{n}\right)
$$

\subsection{Visualisation}

The $2 \mathrm{D}$ coordinates $\boldsymbol{x}_{n}, n=1: N$ provide a visual summary of the data density. In addition, label markers (or colouring information), to aid the visual analysis, are obtained directly from $P_{\boldsymbol{c}_{k}}(k \mid \boldsymbol{x})$. This is a handy feature of our method, as opposed to techniques based on dimensionality reduction methods (such as e.g. PCA), where detecting meaningful clusters from the projection plot is not straightforward. The class labels may also serve as an interface to the domain expert, who may wish to specify and fix the labels of certain points in order to explore the structure of the data interactively.

For accommodating new data points on a visualisation produced from a training set, a fast 'folding in' [6] procedure can be used. This is to compute $\underset{\boldsymbol{x}}{\operatorname{argmax}} p\left(\boldsymbol{d}_{\text {test }} \mid \boldsymbol{x}\right)$ with all model parameters kept fixed to their estimated values. Conveniently, this optimisation task is convex, i.e. with all other parameters kept fixed, the Hessian w.r.t. $\boldsymbol{x}$ is positive semidefinite

$$
\frac{\partial^{2} \mathcal{L}}{\partial \boldsymbol{x}_{n} \partial \boldsymbol{x}_{n}^{T}}=\sum_{k} \sum_{t} r_{k t n} \boldsymbol{c}_{k} \boldsymbol{c}_{k}^{T}-\left\{\sum_{k} \sum_{t} r_{k t n} \boldsymbol{c}_{k}\right\}\left\{\sum_{k} \sum_{t} r_{k t n} \boldsymbol{c}_{k}\right\}^{T}
$$

for the same reasons as in the case of $\mathrm{PE}[7$. Therefore the projection of test points is unique. However, PE [7] makes no mention of how to accommodate new points on an existing visualisation plot.

\subsection{Taking into account estimates of observational error}

It is often the case that data from science domains (e.g. astronomy) come with known observational errors. In this section we modify our algorithm to take these into account. Let $\sigma_{t n}$ be the standard deviation of the known measurement error of the $t$-th feature of instance $n$. We handle this by considering $d_{t n}$ as a hidden variable which stands for the clean data, and in addition we have $\mathcal{N}\left(y_{t n} \mid d_{t n}, 1 / \sigma_{t n}^{2}\right)$.

Assuming that we are dealing with real valued data, $p\left(d_{t n} \mid \boldsymbol{x}_{n}\right)$ was defined as a mixture of Gaussians, and so the integration over the unseen clean data variable $d_{t n}$ gives the following likelihood term for component $k$ of feature $t$ :

$p\left(y_{t n} \mid k\right)=\int d d_{t n} \mathcal{N}\left(y_{t n} \mid d_{t n}, 1 / \sigma_{t n}^{2}\right) \mathcal{N}\left(d_{t n} \mid \mu_{t k}, v_{t k}\right)=\mathcal{N}\left(y_{t n} \mid \mu_{t k},\left(\sigma_{t n}^{2}+1 / v_{t k}\right)^{-1}\right)$ 
In other words, the variance of the data likelihood now has two terms, one coming from the measurement error and one other coming from the modelling error. The latter needs to be estimated.

The estimation equations in this case modify as follows:

$$
r_{k t n}=\frac{\mathcal{N}\left(y_{t n} \mid \mu_{t k},\left(\sigma_{t n}^{2}+1 / v_{t k}\right)^{-1}\right) P_{\boldsymbol{c}_{k}}\left(k \mid \boldsymbol{x}_{n}\right)}{\sum_{k^{\prime}} \mathcal{N}\left(y_{t n} \mid \mu_{t k}, \sigma_{t n}^{2}+1 / v_{t k}\right) P_{\boldsymbol{c}_{k}}\left(k \mid \boldsymbol{x}_{n}\right)}
$$

The update equation of $\mu_{t k}$ becomes

$$
\mu_{t k}=\frac{\sum_{n} y_{t n} r_{k t n} /\left(\sigma_{t n}^{2}+1 / v_{t k}\right)}{\sum_{n} r_{k t n} /\left(\sigma_{t n}^{2}+1 / v_{t k}\right)}
$$

and the updates of $\boldsymbol{x}_{n}$ and $\boldsymbol{c}_{k}$ remain unchanged.

For the precision parameters $v_{t k}$ there is no closed form solution and so numerical optimisation may be employed, e.g. a conjugate gradient w.r.t. $\log v_{t k}$, since then the optimisation is unconstrained.

$$
\frac{\partial}{\partial \log v_{t k}}=\frac{1}{v_{t k}} \sum_{n} r_{k t n}\left\{\frac{1}{2\left(\sigma_{t n}^{2}+1 / v_{t k}\right)}-\frac{\left(d_{t n}-\mu_{t k}\right)^{2}}{2\left(\sigma_{t n}^{2}+1 / v_{t k}\right)^{2}}\right\}-\gamma v_{t k}=0
$$

Observe that when $\sigma_{t n}=0$, all equations of this subsection reduce to those presented for the noise-free case in Sec. 3. Yet another alternative is to treat $d_{t n}$ as hidden variables and take a hierarchical EM approach.

It should be highlighted, that although many non-probabilistic methods simply ignore the measurement errors even when these are known, due to our probabilistic framework a principled treatment is possible. This prevents finding 'interesting' patterns in the visualisation plot as a result of measurement errors, at least in the cases when such errors are known. Furthermore, there are cases when further refinement of the noise model will be needed, e.g. in many cases the recorded error values are uncertain or known to be optimistic.

\section{Experiments and Applications}

\subsection{Numerical simulations}

The first set of experiments is meant to demonstrate the working of our method and to highlight in which situations it is advantageous over the fully disjoint and sequential application of a mixture-based clustering and subsequent visualisation strategy. Illustrative cases are shown and these are important for knowing in what kind of problems is the method appropriate to use.

Throughout, we used smoothness hyperparameters $\alpha=\beta=1$ and $\gamma$ was determined by cross-validation under an initial assumption of a large $(\mathrm{K}=10)$ number of clusters. The priors on the precision parameters favour the extinction of unnecessary components and even if there are remaining redundant components, a good-enough $\gamma$ parameter can be located. The typical value obtained was of the order of $10^{-3}$. Then $\gamma$ is fixed and a further cross-validation is run 
to determine the optimal number of clusters $K$ (less or equal to the number of non-empty clusters found in the previous step). We noted the optimisation is very sensitive to initialisation and starting $\boldsymbol{\mu}_{k}$ from K-means is beneficial. To alleviate problems with local optima, each run was repeated 20 times and the model that found better maximum of the model likelihood was retained for further testing.

Two sets of generated data were created. For the first set, 300 points were drawn from a 6-dimensional mixture of 5 independent Gaussians (60 points in each class). The second set was sampled from a 300-dimensional mixture of 5 independent Gaussians (again, 60 points per class). Fig. 1.a) shows the test likelihood averaged over 20 repeated runs, having generated the test data from the same model as the training data. The test likelihood obtained with a mixture of Gaussians (MoG) is superimposed for comparison. We see that for the relatively low dimensional data set the proposed joint model has little (no) advantage. This is simply because in this case there is enough data to reliably estimate a MoG. The obtained mixture posteriors could then safely be fed into e.g. a PE 7] for class visualisation.

For the case of high dimensional data, however the situation is different. The MoG overfits badly and is therefore unable to identify the clusters or to provide reliable input to a subsequent visualisation method. This is the situation when our proposed model is of use. The projection part of the objective guards against overfitting - as we can see from the test likelihood on Fig 1.b.
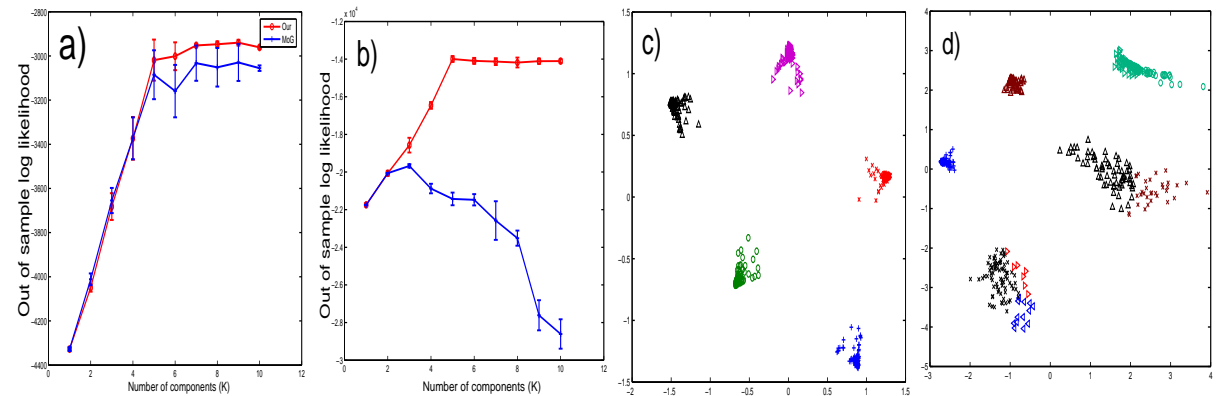

Fig. 1. Experiments on synthetic data: a) Out of sample test likelihood (higher is better) for the 6-dimensional data - our approach vs MoG. b) Same for the 300-dimensional data. Our approach is significantly less prone to overfitting than MoG in this case. c) The final visualisation plot for the 300-dimensional data set. The markers are automatically assigned by the model and in this case they are identical with the true generator classes. d) Illustration of the visualisation process when the number of assumed clusters (ten) is larger than the number of true clusters (five). Notice the true clusters remain compact in the visualisation space. 
Fig 1.c shows the visualisation of the 300-dimensional data set. Each point is the $2 \mathrm{D}$ representation $\left(\boldsymbol{x}_{n}\right)$ of the corresponding $300-\mathrm{D}$ datum point. The markers correspond to the maximum argument of the softmax outputs $P\left(k \mid \boldsymbol{x}_{n}\right)$, so they represent labels automatically assigned by the model. In this case, the estimated labels are identical with the true labels. We also see that the true number of classes has been correctly recovered. In addition, we noted that in experiments where the number of classes was deliberately chosen larger than the true number of clusters, some of the unnecessary clusters progressively become empty indeed, due to the employed prior on the precision parameters, while others split a true cluster. However, notably, the visual image of the true cluster split by several components tends to remain compact. Such an example is seen on Fig 1.d.

\subsection{Visualisation of observed spectra of early-type galaxies}

We apply the method developed above to a sample of measured spectra (in the ultraviolet to optical range of radiation) of 21 well-studied early-type (elliptical or lenticular) galaxies. In a previous work 89, we had studied this data set using various factor analysis techniques. Here, we seek to obtain a visual analysis of the data. Each of these spectra represent flux measurements at 348 values of wavelength in the range $2000-8000 \AA$, in equal bins, for all spectra. Observational errors are associated with each value, which we take into account as described in Section 3.3. Thus, the clustering and class visualisation of these spectra is a high-dimensional problem.

This represents a pilot data set for an important study in the evolution of galaxies. It is generally believed that all early-type galaxies formed all their stars in the early universe, and then have evolved more-or-less passively until the present day- so one expects to find their spectrum to correspond to a collection of stars all of the same age. However, detailed observations in the last decade indicate a wealth of complex detail in a significant fraction of such galaxies, including evidence of a sub-population of very young stars in many cases. How common this effect is largely unknown, and can only be addressed through data mining of large spectral archives. Even though many $\times 10^{5}$ galaxy spectra are being assembled in large public archives (e.g. www.sdss.org), a sample as detailed as ours is rare and difficult to assemble, particularly with such wide a coverage in wavelength, which requires combining observations from both ground and space based observatories (see details in 9]). From this small sample, we would attempt to isolate those galaxies which have young stars from those that don't.

Needless to say, the fluxes are all positive values. In order to be interpretable, our method needs to ensure the estimated parameters (cluster prototypes) are also positive. In our previous work, we built in explicit constraints to ensure this 9]. Here, since each $\mu_{t k}$ in (11) is just a weighted average of positive data, its positivity is automatically satisfied.

The leave-one-out test likelihood of our model is shown on the left plot of Fig. 2. The peak at $K=2$ indicates that two clusters have been identified. A mixture of Gaussians test likelihood is superimposed for comparison, showing 
the overfitting problem due to the high dimensionality. The MoG is therefore unable to identify any clusters in the data. Hence, a class visualisation of the data based on mixture posteriors would be clearly compromised in this case. The right hand plot shows the grouping of the actual data around the two identified prototypes $\boldsymbol{\mu}_{k}, k=1,2$ of our model. The latter are superimposed with thick lines. These can be recognised and interpreted as the prototype of the spectrum of a 'young' and 'mature' stellar population respectively. Thus, in this case, the clusters have a physical interpretation in astrophysical terms.
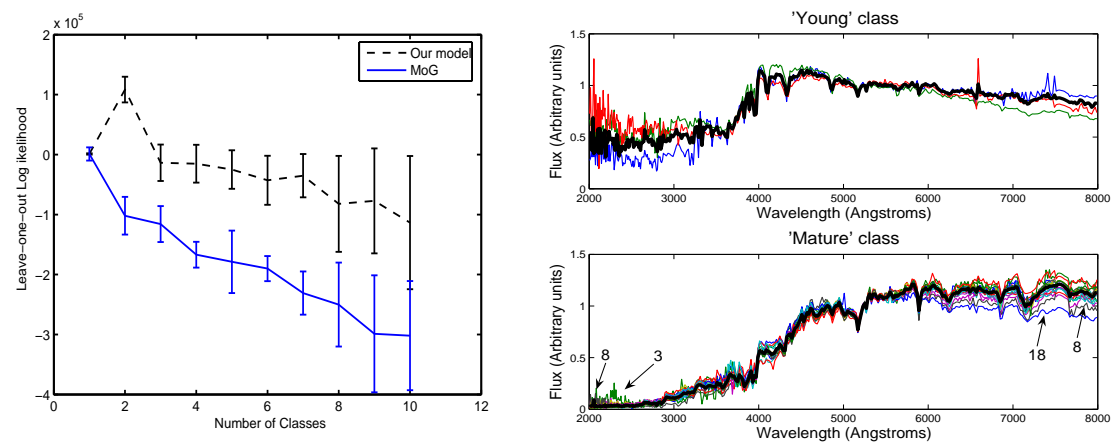

Fig. 2. Left: The leave-one-out test likelihood versus the number of clusters. The peak at $K=2$ produced by our method indicates two clusters whereas a mixture of Gaussians overfits and therefore fails to identify clusters in the data. Right: The actual data, clustered around the two prototypes identified by our model. The parameters $\boldsymbol{\mu}_{k}$ for $k=1,2$ are superimposed with thick lines. They are interpretable as a 'young' and an 'old' prototypical spectrum respectively. The identification number that marks some of the spectra correspond to those on Fig. 3. The marked spectra are the instances that apart from their overall shape present some features of the 'young' category too.

Identification numbers mark some of the spectra clustered in the 'mature' category on the left lower plot of Fig. 2. These are the galaxies that have a significantly non-zero class membership for either cluster, and they indeed include some morphological aspects of the 'young' category as well (the emission lines at $<2000 \AA$ and the slope of the spectral continuum in the range 6000 $8000 \AA)$. Physically, this indicates the presence of a significant population of young $(<2$ Gyr old) stars, whereas the rest of the stars are $>10$ Gyr old.

The identification numbers are the same as those on Fig. 3, where we see the $2 \mathrm{D}$ visualisation of the sample on the left. For each spectrum $\boldsymbol{y}_{n}$, the $2 \mathrm{D}$ latent representation $\boldsymbol{x}_{n}$ is plotted. The markers represent cluster labels automatically assigned by the model, as detailed in the right hand plot. We see the two clusters are well separated on the image and the 'hybrid' galaxies are indeed placed in 

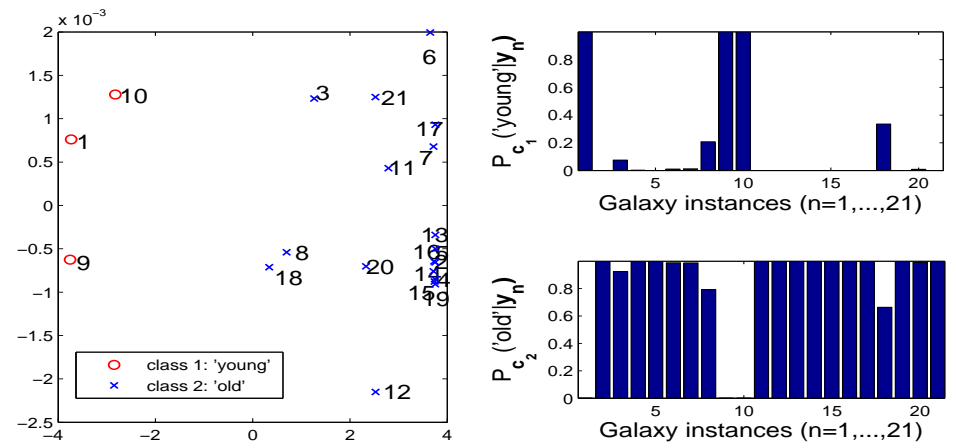

Fig. 3. Left: Visualisation of the data set of the spectra of 21 early-type galaxies. For each spectrum $\boldsymbol{y}_{n}$, the $2 \mathrm{D}$ latent representation $\boldsymbol{x}_{n}$ is plotted. The markers are those assigned by the model, as shown on the right.

between those that are clearly cluster members. Of these, the one marked as 18 represents a galaxy (NGC 3605) for which recent detailed physical analyses have been made 10. It turns out that although more than $85 \%$ of its stellar mass is associated with an old (9-12 Gyr) stellar population, it does contain a younger stellar population too, at $\simeq 1$ Gyr $[10$.

We therefore conclude that it is possible to have an intuitive visual summary of a few hundreds of measurements per galaxy in just two coordinates with the application of our method.

\subsection{Visual analysis of gene expressions}

In a brief final experiment we show the potential use of our approach for the visual analysis of high dimensional gene expression arrays. Oligonucleotide arrays can provide a means of studying the state of a cell, by monitoring the expression level of thousands of genes at the same time [1] and have been the focus of extensive research. The main difficulty is that the number of examples is typically of the order of tens while the number of genes is of the order of thousands. Even after eliminating genes that have little variation, we are still left with at least hundreds of data dimensions. Straightforward mixture based clustering runs into the well-know curse of dimensionality problem. Here we apply our method to the ColonCancer data set, having 40 tumour and 22 normal colon tissue samples 1 . This is a benchmark data set, used in many previous classification and clustering studies. Our input matrix consisted of the 500 genes with highest overall variation $\times$ the 62 samples.

Fig. 4 shows the visualisation obtained in a purely unsupervised manner. The markers now correspond to the true labels (not used by the algorithm), and are given for the ease of visual evaluation of the representation produced. The separation of cancerous from noncancerous tissues is most apparent on the plot. 
The potential of such a visualisation tool lies mainly in that it would allow a domain expert to interactively explore the structure of the sample. Additionally, the gene-specific posteriors $r_{k t n}$ provide quantitative gene-level class information.

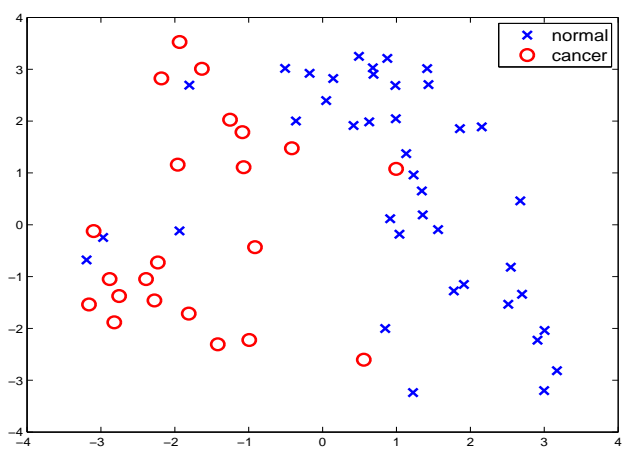

Fig. 4. Unsupervised class visualisation of the ColonCancer data set. The markers correspond to the true class for the ease of visual evaluation.

\section{Conclusions}

We proposed and investigated a model for class visualisation of explicitly high dimensional data. We have shown this model relates closely to PE [7 in that it represents a probabilistic integration of the clustering and visualisation objectives into a single model. We derived empirical Bayesian estimates for our model which make this multi-objective interpretation easy to follow. Although this work may potentially further be enhanced by a fuller Bayesian estimation scheme, the empirical Bayesian methodology has been appropriate for our purposes [11] and it allows us to estimate an empirical latent density from a given example set of data and to reason about previously unseen data relative to that. We demonstrated gains in terms of the predictive capabilities of the proposed model over the fully modular and sequential approach to clustering and class visualisation in the case of high dimensional data.

Acknowledgements This research is funded by PPARC grant PP/C503138/1, 'Designer Algorithms for Astronomical Data Mining'. AK also acknowledges partial support from a Wellcome Trust VIP Award (Project 10835). 


\section{Appendix. Estimation of $\boldsymbol{x}_{n}$}

The terms containing $\boldsymbol{x}_{n}$ are the following.

$$
\begin{aligned}
& \sum_{n} \sum_{t} \sum_{k} r_{k t n}\left\{-\frac{1}{2}\left(\boldsymbol{x}_{n}-\boldsymbol{c}_{k}\right)^{2}-\log \sum_{k^{\prime}} \exp \left(-\frac{1}{2}\left(\boldsymbol{x}_{n}-\boldsymbol{c}_{k}\right)^{2}\right)\right\}-\alpha \boldsymbol{x}_{n}^{2} \\
= & \sum_{n} \sum_{t}\left\{-\sum_{k} r_{k t n} \frac{1}{2}\left(\boldsymbol{x}_{n}-\boldsymbol{c}_{k}\right)^{2}-\log \sum_{k^{\prime}} \exp \left(-\frac{1}{2}\left(\boldsymbol{x}_{n}-\boldsymbol{c}_{k}\right)^{2}\right)\right\}-\alpha \boldsymbol{x}_{n}^{2}
\end{aligned}
$$

The gradient is then:

$$
\frac{\partial}{\partial \boldsymbol{x}_{n}}=\sum_{t}\left\{-\sum_{k} r_{k t n}\left(\boldsymbol{x}_{n}-\boldsymbol{c}_{k}\right)+\sum_{k^{\prime \prime}} \frac{\exp \left(-\frac{1}{2}\left(\boldsymbol{x}_{n}-\boldsymbol{c}_{k^{\prime \prime}}\right)^{2}\right)}{\sum_{k^{\prime}} \exp \left(-\frac{1}{2}\left(\boldsymbol{x}_{n}-\boldsymbol{c}_{k^{\prime}}\right)^{2}\right)}\left(\boldsymbol{x}_{n}-\boldsymbol{c}_{k^{\prime \prime}}\right)\right\}-\alpha \boldsymbol{x}_{n}
$$

Renaming $k^{\prime \prime}$ by $k$ and replacing the expression of $P \boldsymbol{c}_{k}\left(k \mid \boldsymbol{x}_{n}\right)$ the following is obtained.

$$
\begin{aligned}
\frac{\partial}{\partial \boldsymbol{x}_{n}} & =\sum_{t} \sum_{k}\left\{-r_{k t n}\left(\boldsymbol{x}_{n}-\boldsymbol{c}_{k}\right)+P \boldsymbol{c}_{k}\left(k \mid \boldsymbol{x}_{n}\right)\left(\boldsymbol{x}_{n}-\boldsymbol{c}_{k}\right)\right\}-\alpha \boldsymbol{x}_{n} \\
& =\sum_{k}\left(\boldsymbol{c}_{k}-\boldsymbol{x}_{n}\right) \sum_{t}\left(r_{k t n}-P \boldsymbol{c}_{k}\left(k \mid \boldsymbol{x}_{n}\right)\right)-\alpha \boldsymbol{x}_{n}
\end{aligned}
$$

\section{References}

1. U Alon, N Barkai, D Notterman, K Gish, S Ybarra, D Mack, A Levine. Broad Patterns of Gene Expression Revealed by Clustering Analysis of Tumour and Normal Colon Cancer Tissues Probed by Oligonucleotide Arrays. Cell Biol. 96, 6745-6750.

2. H Attias. Learning in High Dimension: Modular mixture models. Proc. Artificial Intelligence and Statistics, 2001.

3. C.M Bishop. Neural Networks for Pattern Recognition. Oxford University Press, Inc., New York, NY, 1995.

4. C.M Bishop, M Svensen and C.K.I Williams. GTM: The Generative Topographic Mapping. Neural Computation, vol. 10(1), 1998.

5. B.P Carlin and T.A Louis. Bayes and Empirical Bayes Methods for Data Analysis. Chapman and Hall, 2000.

6. Th Hofmann. Gaussian Latent Semantic Models for Collaborative Filtering. 26th Annual International ACM SIGIR Conference, 2003.

7. T Iwata, K Saito, N Ueda, S Stromsten, T.L Griffiths, J.B Tenenbaum. Parameteric Embedding for Class Visualisation. Proc. Neur. Information Processing Systems 17, 2005.

8. A Kabán, L Nolan and S Raychaudhury. Finding Young Stellar Populations in Elliptical Galaxies from Independent Components of Optical Spectra. Proc. SIAM Int'l Conf on Data Mining (SDM05), pp. 183-194.

9. L Nolan, M Harva, A Kabán and S Raychaudhury. A data-driven Bayesian approach to finding young stellar populations in early-type galaxies from their ultraviolet-optical spectra, Mon. Not. of the Royal Astron. Soc. 366,321-338, 2006.

10. L Nolan, J.S Dunlop, B Panter, R Jimenez, A Heavens, G Smith. The starformation histories of elliptical galaxies across the fundamental plane, submitted to MNRAS. 
11. J Rice. Reflections on SCMA III. In: Statistical challenges in astronomy. Eds: E.C Feigelson and G.J Babu. Springer. 2003.

12. S Rogers, M Girolami, C Campbell, R Breitling. The latent process decomposition of cDNA microarray datasets. IEEE/ACM Transact. Comput. Biol. Bioinformatics. 2: 143-156.

13. T Soukup and I Davidson. Visual Data Mining: Techniques and Tools for Data Visualisation and Mining. Wiley, 2002. 